\title{
USING NEURAL NETWORKS TO IDENTIFY DRIVING STYLE AND HEADWAY CONTROL BEHAVIOR OF DRIVERS
}

\author{
C. MacADAM, Z. BAREKET, P. FANCHER, and R. ERVIN I
}

\begin{abstract}
SUMMARY
This paper illustrates the use of neural network techniques for analyzing headway data collected from a group of 36 driving subjects during normal on-highway driving. Pattern recognition methods are used to identify different types of headway-keeping behavior exhibited by these drivers and their relative distributions. Possibilities for using neural networks to represent longitudinal control behavior of drivers are also considered and discussed.
\end{abstract}

\section{INTRODUCTION}

Recent studies $[1,2,3,4]$ examining the possible introduction of headway control systems for selected highway vehicles have intensified interest in the longitudinal control behavior of actual drivers. Much of this interest is focused on better understanding the range of driving styles present in the highway traffic population so as to smoothly bridge and match the control characteristics of emerging headway control technologies to preferences exhibited by human drivers. Such preferences normally include items like: acceleration/deceleration comfort levels, headway gap sizes employed during following, and overall level of aggressiveness related to passing and overtaking behavior.

This paper illustrates two uses of neural networks for: 1) identifying and classifying on-highway longitudinal control behavior of drivers based on different levels of displayed aggressiveness, and 2) representing or modelling instances of longitudinal control behavior for potential use in ITS headway control system algorithms. Recent measurements [2] of on-highway driving behavior for a group of 36 lay drivers (and their corresponding vehicle response measurements) are used to conduct the analyses.

One motivation for using a neural network approach for behavior classification stems from the desire to conduct efficient searches of various types of driving behavior located within relatively large volumes of stored time history data. The pattern recognition ability of certain neural network architectures is well known and lends itself well to this type of task [6]. The resulting information can be used in a variety of applications ranging from human factors studies concerned with distributions of ordinary driver control behavior, to uses within headway control systems. In the latter application, headway control system parameters could be assigned different values based upon observed categories of driver behavior identified by an on-board neural net processor.

A second application illustrates how neural networks can be used to also represent or model driver throttle control responses during close-following conditions. This type

1 The University of Michigan Transportation Research Institute 
of application is focused more on real time, on-line identification of driver longitudinal control behavior. Its use as an adaptive control modelling technique can have utility within the context of warning/control packages that require a continuously updated characterization of driver behavior. It might also be used as an adaptive control algorithm within a headway control system, replacing the functionality of a traditional controller.

The paper begins with the pattern recognition example - demonstrating the utility of neural nets in identifying and categorizing various types of observed longitudinal control behavior. The control modelling example follows.

\section{CATEGORIZING DRIVING BEHAVIOR - METHODOLOGY}

The following analyses utilize a databank of measurements from an on-going study of forward crash avoidance systems [2] covering a group of 36 drivers. Each driver operated the same test vehicle under normal highway conditions in the U.S. The same itinerary was followed by each driver and consisted of four separate freeways connected by interchange loops. The general location was the western Detroit suburban area. Each trip lasted about one hour in duration. Driver/vehicle responses were recorded and stored on-board. Measurements included: range and range-rate provided by a Leica infrared forward-looking sensor; driver steering. braking, and throttle control activity; and other vehicle responses such as yaw rate and lateral acceleration. This paper addresses only the manual driving trips during which the headway throttle control system was inactivated, but range and range-rate were still recorded.

The collected data were then processed by neural net methods designed to efficiently search for and categorize certain types of driving behavior. Classification of driving style grouped drivers according to their displayed level of aggressiveness as defined here by a willingness to pass, follow, or be passed by other vehicles. Five categories of driving behavior, based upon relative rates of longitudinal closure, were defined to represent differing levels of aggressivity. A neural network was then developed and trained to automatically identify and classify these observed patterns into the appropriately defined driving style categories. The inputs to the neural network were range and range rate (for vehicle speeds above $40 \mathrm{mph}$ ). The network output is the classification of driving style based upon observed input patterns occurring in the data stream. A 10-second moving window was used for each evaluation. See Figure 1.

Each of the five output categories represents the degree to which the host vehicle either closed-in on or fell behind adjacent vehicles during ordinary driving. The middle category, entitled "following." is reflective of constant speed / constant headway driving behavior that most drivers utilize most of the time.

The neural network architecture used for this pattern recognition problem is seen in Figure 2. It contains 40 input nodes (corresponding to 20 samples each of range and range-rate measurements), 9 first-layer neurons, and 6 output-layer neurons (each 
associated with one of the 5 categories of driving behavior, plus a "no target" category).

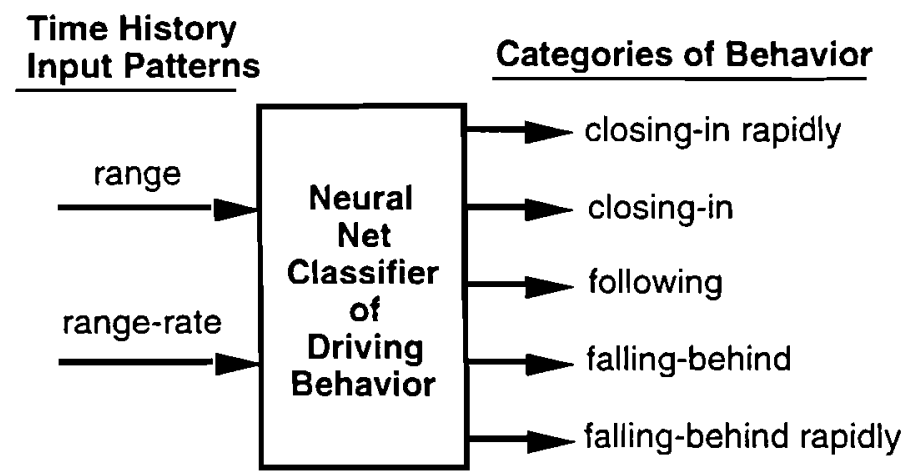

Fig. 1. Neural net pattern recognition for classifying driving behavior.

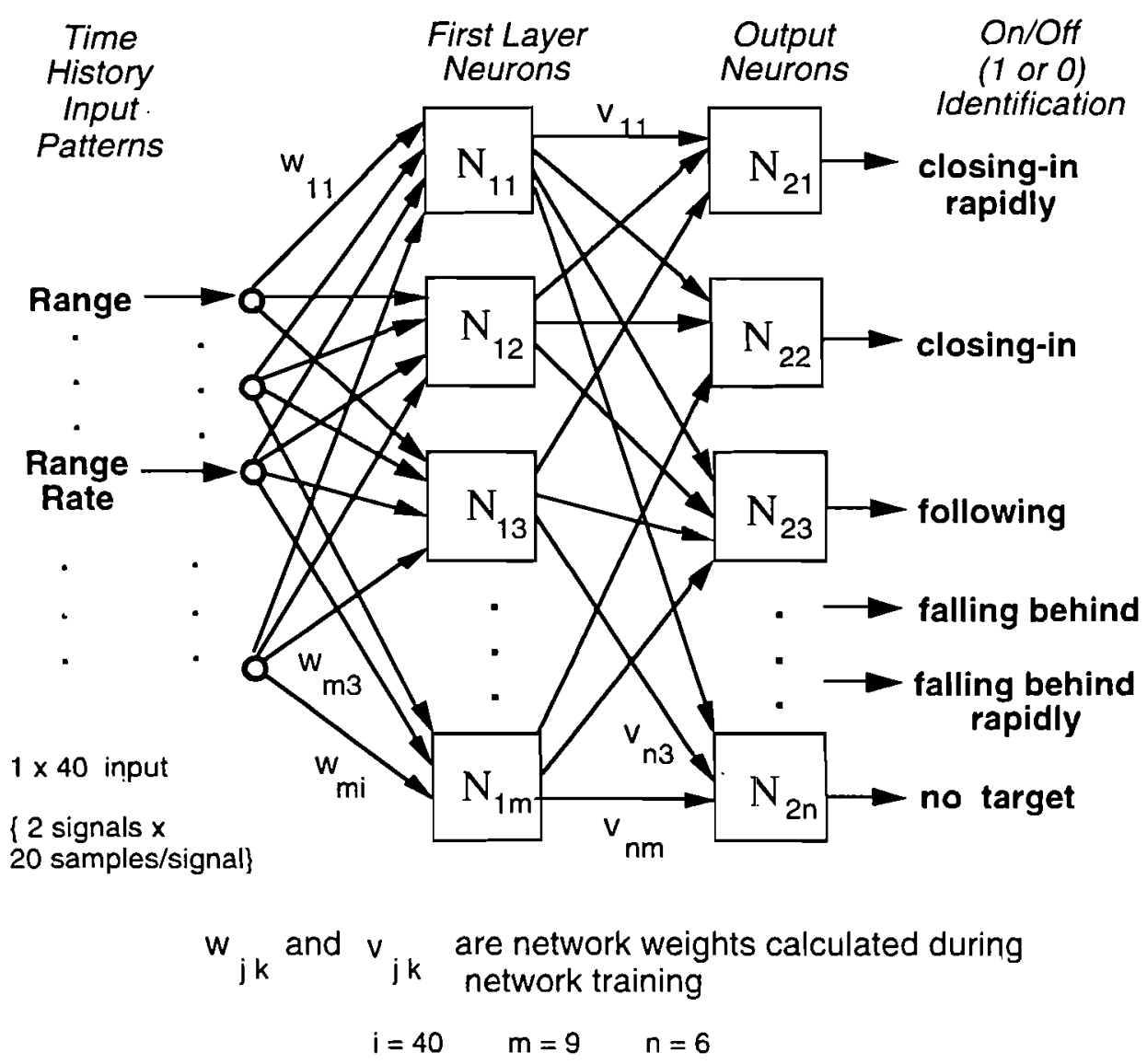

Fig. 2. Neural net architecture used for classifying driving behavior. 
A nonlinear sigmoid, defined in Figure 3, defines each neuron's activation function in the first layer. The output layer neurons are linear. At the output layer, a neuron value of 1 , coupled with all other output neurons at zero, is defined as a recognition condition for that neuron containing the value of 1 . For example, if the first output neuron is associated with the "closing-in rapidly" behavior category, then its activation at 1 , and all others at 0 , indicate the presence of a "closing-in rapidly" event for the current input pattern.

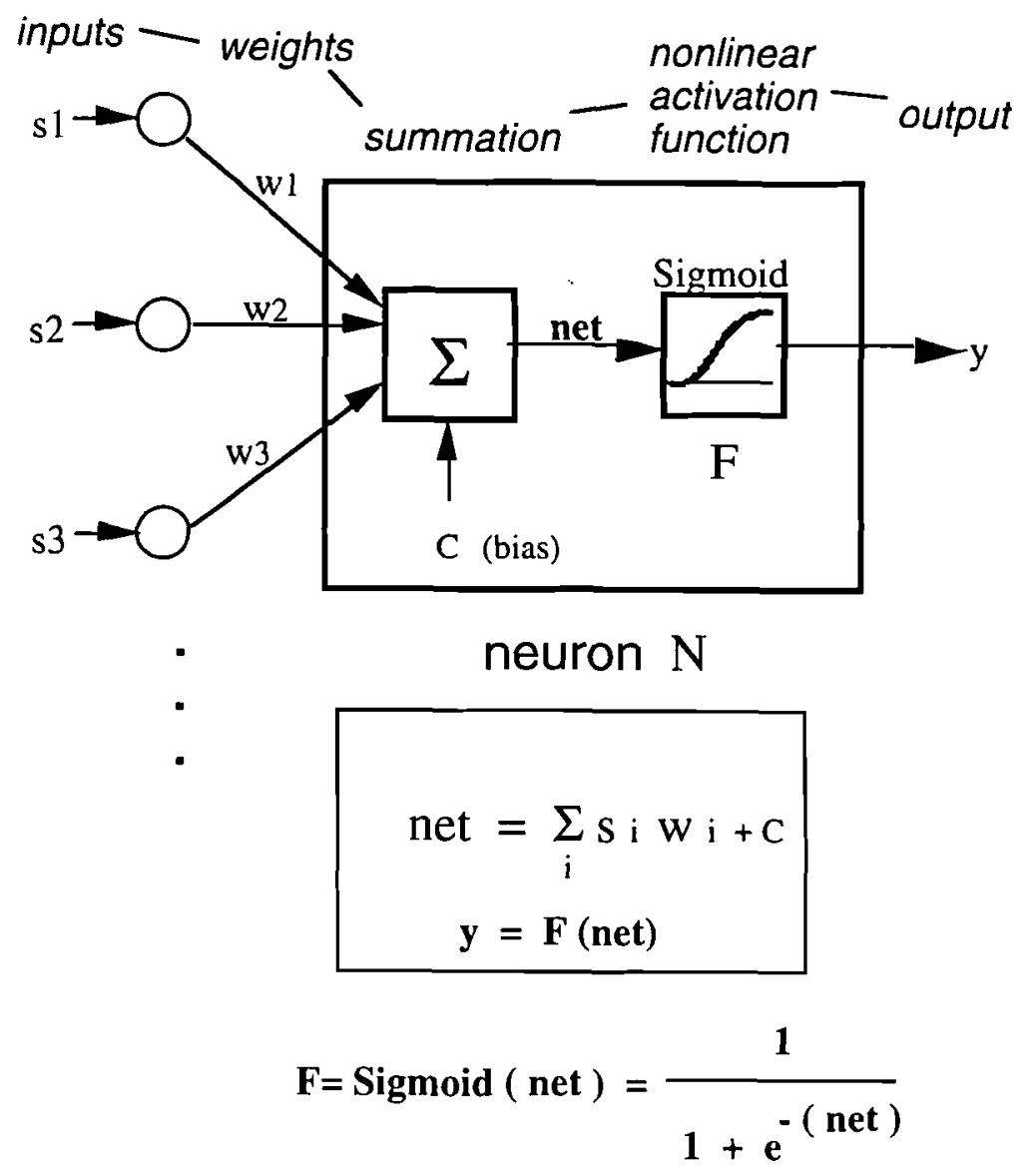

Fig. 3. Neuron element definition.

Training of the network involves an iterative process (back-propagation [6]) by which pairs of input patterns and corresponding output training patterns are simultaneously presented to the network. For each input pattern presented to the network, a corresponding training pattern (comprised of 0 's and a single 1 located at the neuron intended to be associated with that input pattern) is also required at the output layer. Network weight values are then iteratively adjusted until output errors (differences 
between what the network calculates at its output layer and the corresponding value of its output training pattem) are minimized. The process of network training produces a set of weight values that then defines, in conjunction with the network architecture, the pattern recognition algorithm. This can then be used to process other input patterns, heretofore unseen, that are similar in nature to those input patterns used during the network training.

Figure 4 contains five sets (a-e) of training inputs used in this application obtained from 4 of the 36 driving subjects. Each input pattern consists of two contiguous sets of range and range-rate time history measurements. Each time history is 20 samples long with a sampling rate of $2 \mathrm{~Hz}$ (or 10 seconds duration). Approximately 12-15 example training patterns were manually selected and used to define each of the five categories (appearing below as figures $4 \mathrm{a}-4 \mathrm{e}$ ). The range and range-rate data appearing in Figure 4 are normalized in units of feet and feet per second. Range is defined as the distance from the lead (target) vehicle to the host (trailing) vehicle.

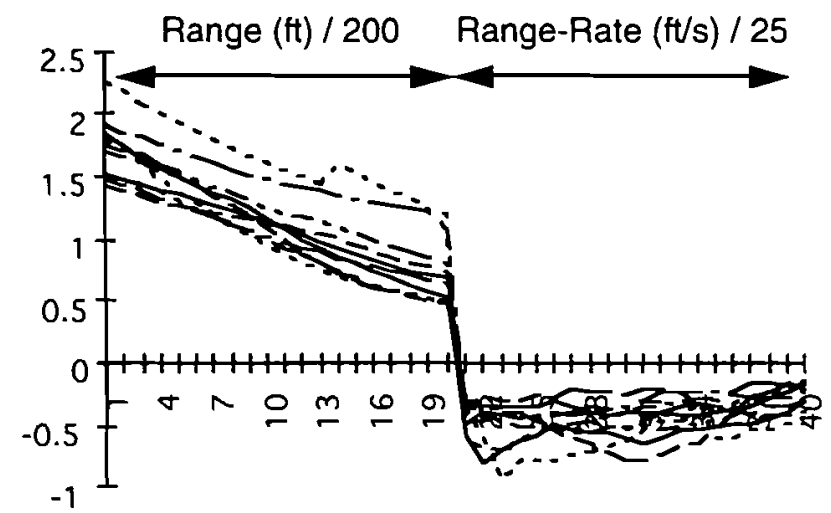

Fig. 4a. Input patterns used to train the neural net - Closing In Rapidly.

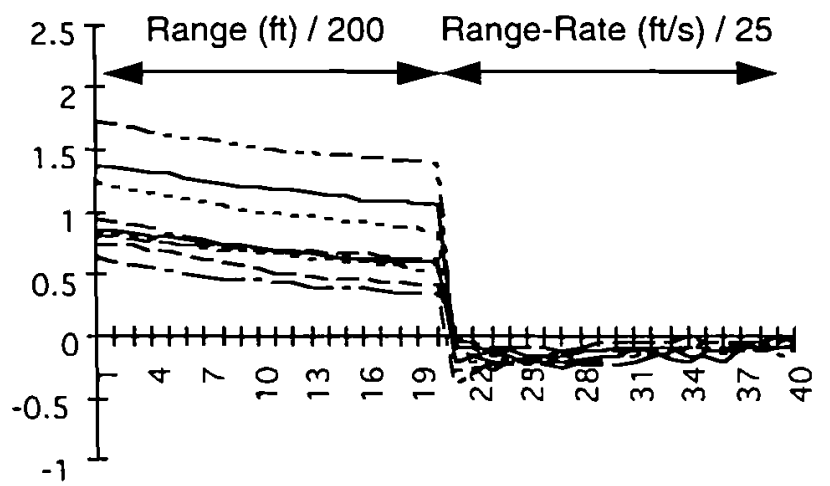

Fig. 4b. Input patterns used to train the neural net - Closing In. 


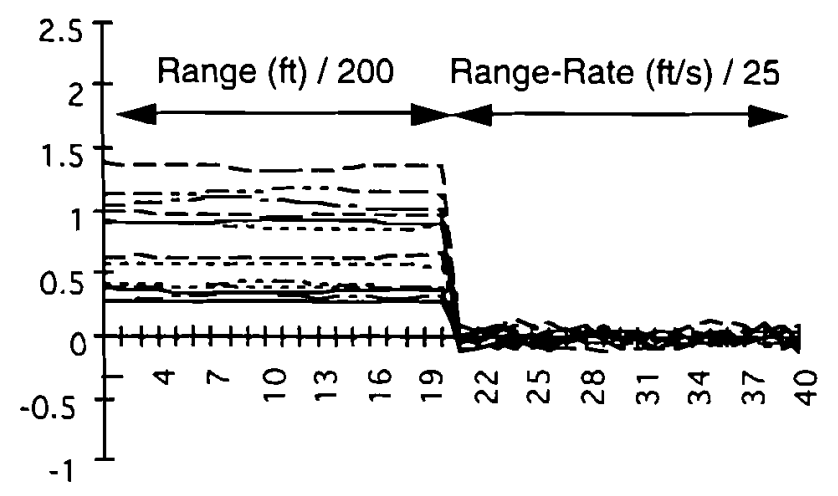

Fig. 4c. Input patterns used to train the neural net - Following.

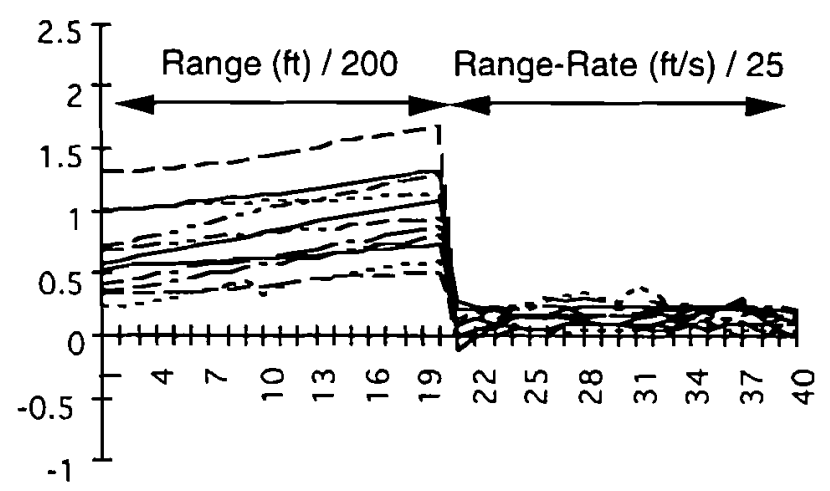

Fig. 4d. Input patterns used to train the neural net - Falling Behind.

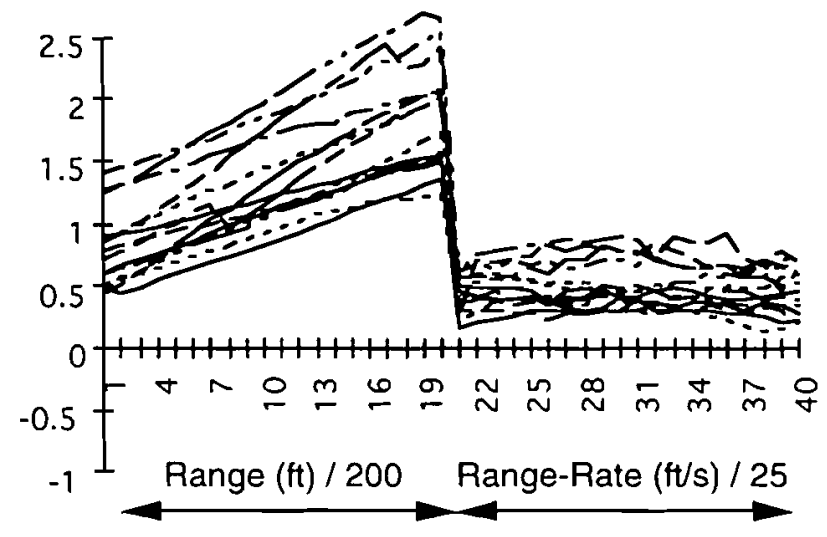

Fig. 4e. Input patterns used to train the neural net - Falling Behind Rapidly.

Following the network training using the data of Figure 4, the resulting algorithm (network weights and architecture of Figure 2) was then applied to the entire database of 
all 36 drivers. For each driver, a tabulation was made of (a) input patterns recognized by the neural net processor as recognizable, (b) non-recognizable data, and (c) cases where no sensor target was present. This information was then summarized in histograms and is described further in the next section.

\section{RESULTS FOR ALL 36 DRIVERS}

By applying the above analysis to each of the 36 drivers, individual as well as cumulative distributions of the five driving behavior categories were obtained. An example result for a fairly "average" driver is seen in Figure 5. The data for this driver (subject \#7) indicate a relatively normal distribution of elected passing behavior. The willingness to pass other vehicles is more or less balanced by a willingness to be passed or to follow. Passing engagements constituted $38 \%$ of the encounters; following occurred $33 \%$ of the time; and for the remaining $29 \%$ of the encounter time, this subject was passed by other vehicles.

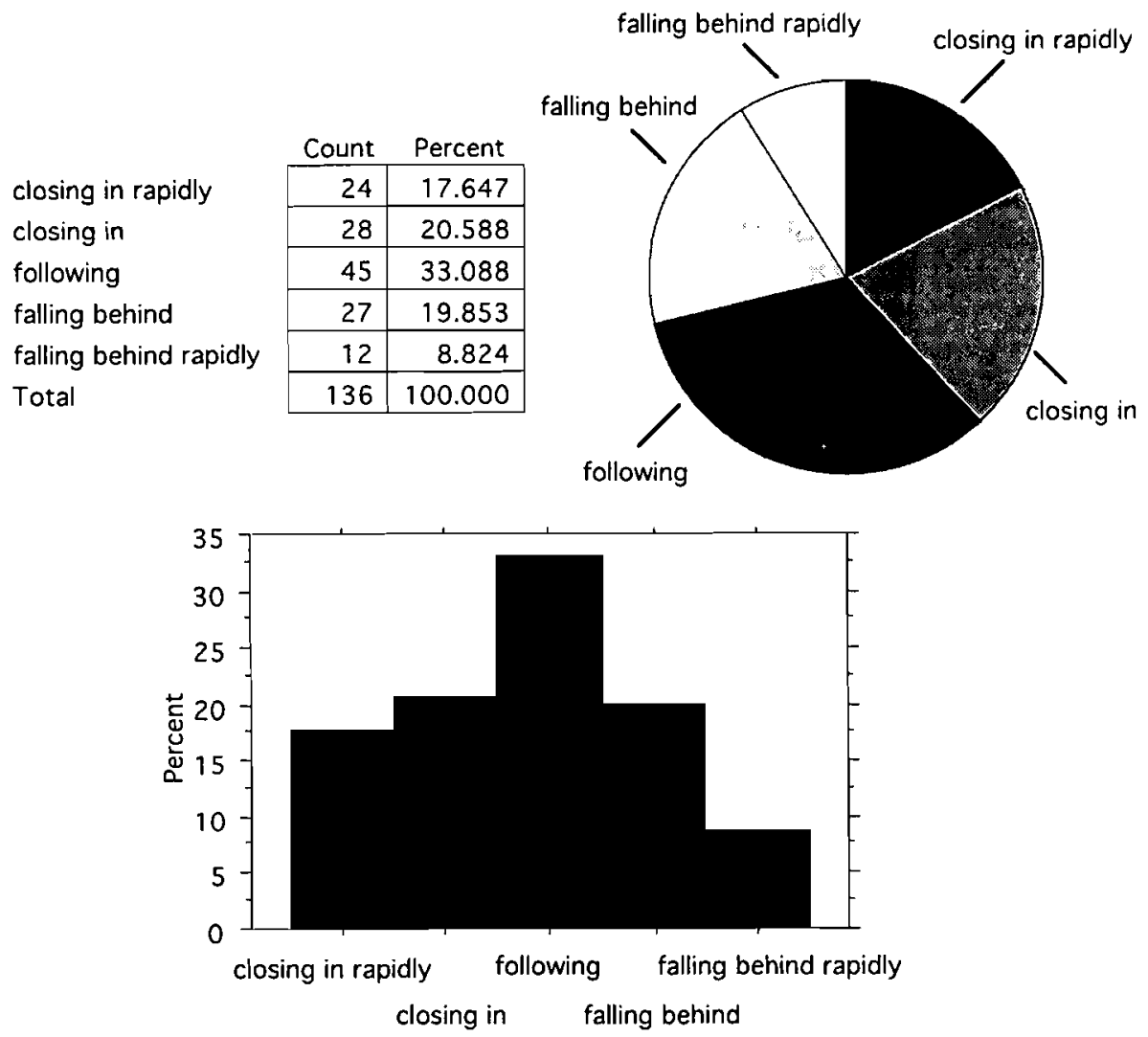

Fig. 5. Distribution of driving behavior identified for an "average" driver over a 1 -hour period. 
Also for this driver, for the total time spent on the 1-hour trip, $46 \%$ of the data constituted recognizable driving scenarios (as defined by one of the five categories), $18 \%$ of the time no valid range sensor target was present (i.e., no target vehicle was in the detection range of the sensor), and the remaining $36 \%$ of data were non-recognizable scenarios (e.g., vehicles cutting in and out of lanes for short periods of time, or intermittent sensor signals having no particular recognizable quality).

By way of contrast, the data for subject \#1 seen in Figure 6, indicate a much greater tendency to pass other vehicles than to be passed. During encounters with other vehicles, this subject engaged in overtaking maneuvers more than $50 \%$ of the time, but was passed by other vehicles less than $10 \%$ of the time. The remaining $40 \%$ of encounter time was spent following another vehicle.

Similar to the previous "average" driver, of the total time spent on the 1-hour trip, $46 \%$ of the data constituted recognizable driving scenarios, $15 \%$ of the time no valid range sensor target was present, and the remaining $39 \%$ of data were non-recognizable scenarios.
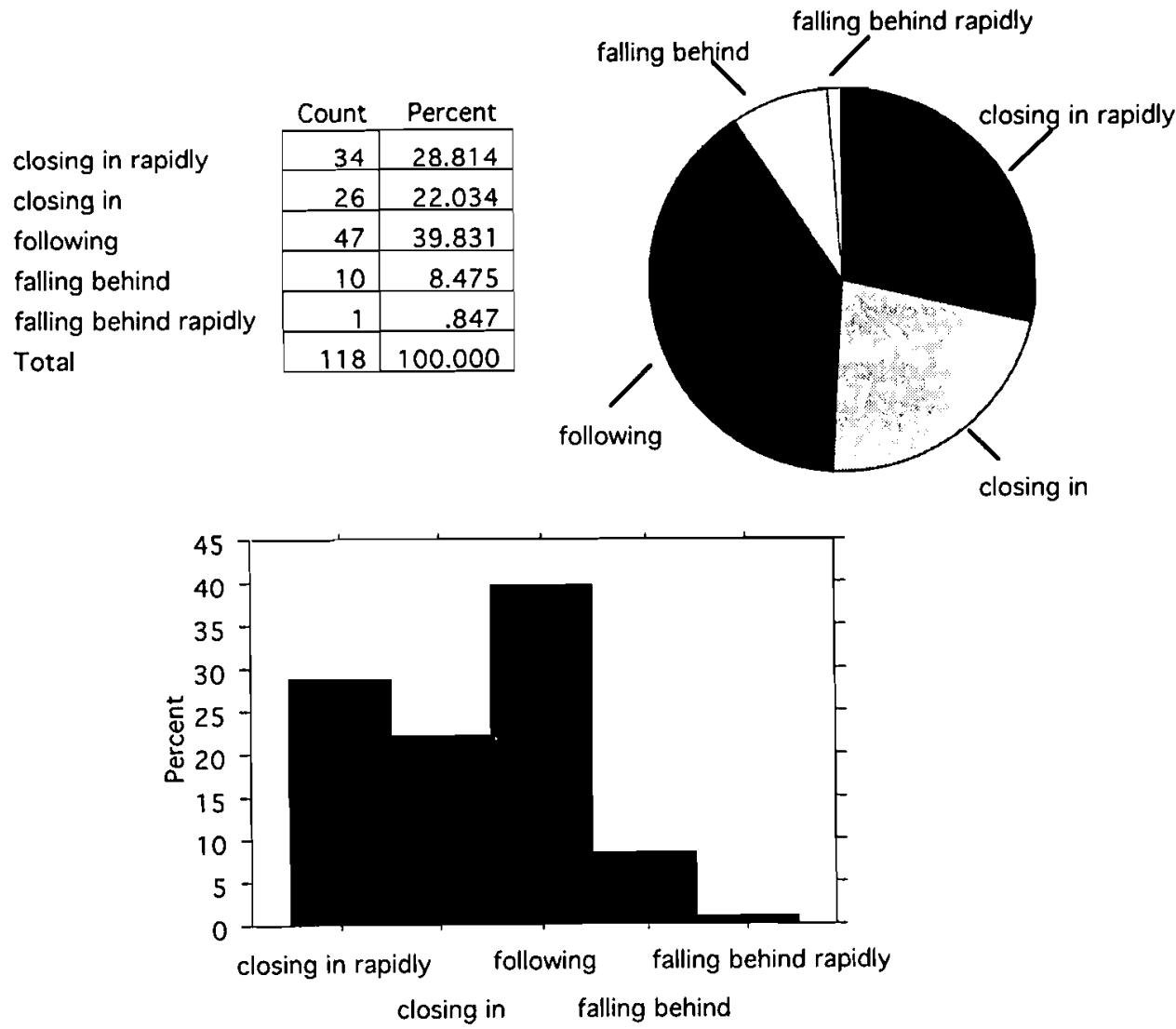

Fig. 6. Distribution of driving behavior identified for a more aggressive driver. 
At the other end of the scale, the data for subject \#13 in Figure 7 show a much reduced willingness to pass other vehicles (10\% of the encounter time). Another $10 \%$ of the encounter time was spent following other vehicles. The remaining $80 \%$ of the encounter time was spent being passed by other vehicles.

Of the total trip time, $39 \%$ constituted recognizable driving scenarios (versus $46 \%$ and $46 \%$ respectively for the previous two drivers); $19 \%$ of the time no valid target was present (versus $18 \%$ and $15 \%$ ); and the remaining $42 \%$ was deemed non-recognizable data (versus $36 \%$ and $39 \%$ ).
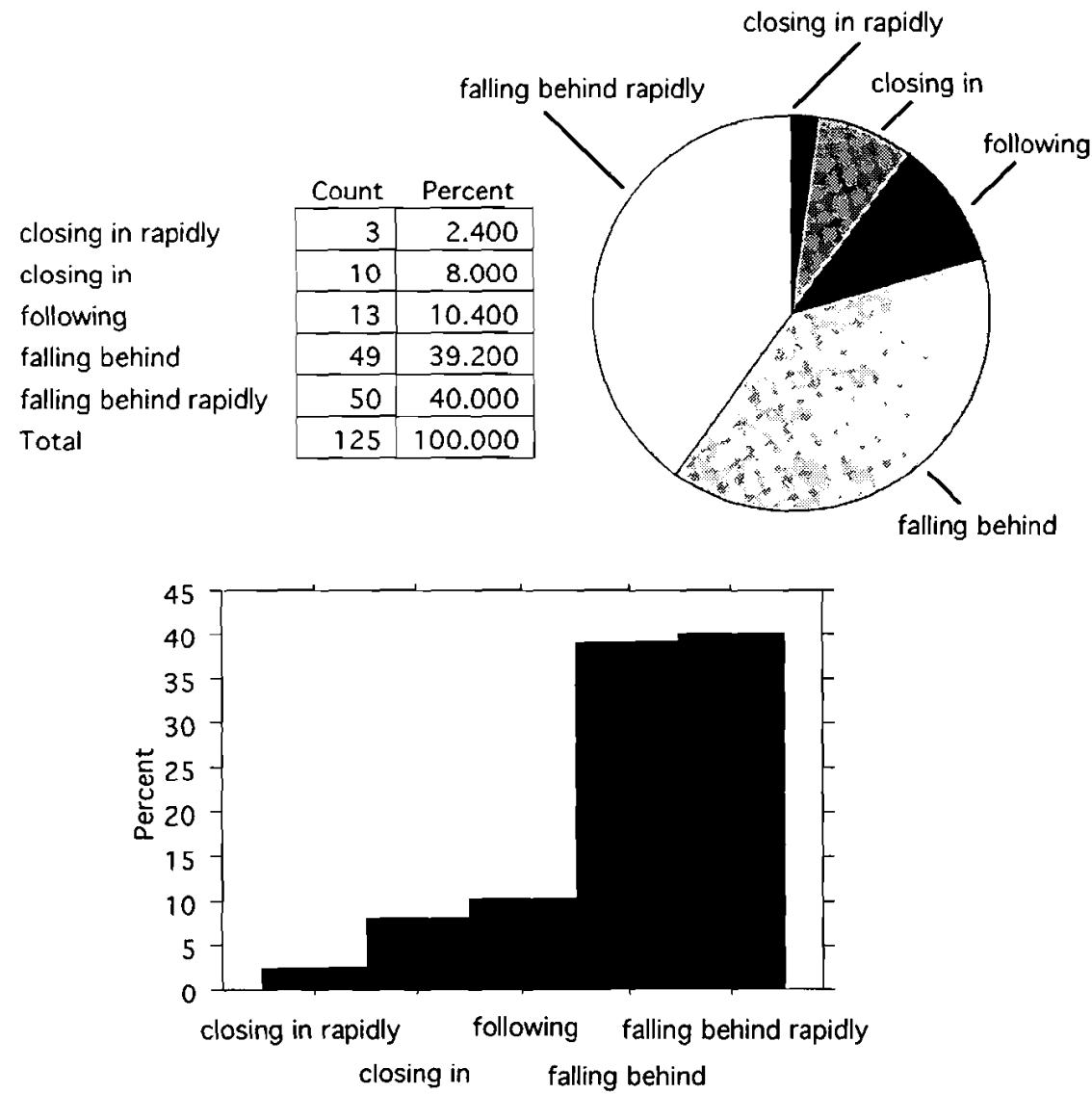

Fig. 7. Distribution of driving behavior identified for a more passive driver.

Table 1 summarizes similar results for all 36 driving subjects. The first column contains the identification number of the driver. The next 5 columns contain the percentage of time each of the five driving behavior categories/scenarios was recognized as valid data by the neural net processor. Columns $7-9$ pertain to the total trip time with column 7 showing what percentage of the total time was deemed recognizable by 
Table 1. Summary of driving behavior results for all 36 drivers.

\begin{tabular}{|c|c|c|c|c|c|c|c|c|}
\hline $\begin{array}{c}\text { Subject } \\
\#\end{array}$ & $\begin{array}{l}\text { Closing- } \\
\text { in } \\
\text { rapidly } \\
\%\end{array}$ & $\begin{array}{c}\text { Closing-in } \\
(\%)\end{array}$ & $\begin{array}{l}\text { Following } \\
\qquad(\%)\end{array}$ & $\begin{array}{l}\text { Falling } \\
\text { behind } \\
(\%)\end{array}$ & $\begin{array}{l}\text { Falling } \\
\text { behind } \\
\text { rapidly } \\
(\%)\end{array}$ & $\begin{array}{c}\text { Recognize-I } \\
\text { Driving } \\
\text { Scenarios } \\
(\%)\end{array}$ & $\begin{array}{l}\text { No } \\
\text { Target } \\
(\%)\end{array}$ & $\begin{array}{c}\text { Non- } \\
\text { Recognize-I } \\
\text { Data } \\
(\%)\end{array}$ \\
\hline 1 & 29 & 22 & 40 & 8 & 1 & 46 & 15 & 39 \\
\hline 2 & 14 & 27 & 35 & 18 & 6 & 53 & 2 & 45 \\
\hline 3 & 30 & 22 & 24 & 19 & 4 & 39 & 23 & 38 \\
\hline 4 & 10 & 20 & 28 & 35 & 7 & 64 & 4 & 32 \\
\hline 5 & 18 & 34 & 25 & 18 & 5 & 56 & 2 & 42 \\
\hline 6 & 22 & 20 & 18 & 32 & 8 & 49 & 16 & 35 \\
\hline 7 & 18 & 20 & 33 & 20 & 9 & 46 & 18 & 36 \\
\hline 8 & 21 & 31 & 37 & 9 & 2 & 46 & 17 & 37 \\
\hline 9 & 11 & 13 & 30 & 23 & 23 & 45 & 18 & 37 \\
\hline 10 & 11 & 24 & 24 & 30 & 11 & 54 & 14 & 32 \\
\hline 11 & 17 & 29 & 43 & 10 & 1 & 67 & 4 & 29 \\
\hline 12 & 38 & 27 & 26 & 9 & 0 & 49 & 11 & 40 \\
\hline 13 & 3 & 8 & 10 & 39 & 40 & 39 & 19 & 42 \\
\hline 14 & 11 & 23 & 40 & 25 & 1 & 60 & 5 & 35 \\
\hline 15 & 10 & 21 & 24 & 18 & 27 & 47 & 11 & 42 \\
\hline 16 & 16 & 25 & 30 & 26 & 3 & 61 & 3 & 36 \\
\hline 17 & 10 & 25 & 15 & 29 & 21 & 29 & 28 & 43 \\
\hline 18 & 12 & 26 & 27 & 28 & 7 & 53 & 11 & 34 \\
\hline 19 & 9 & 15 & 26 & 26 & 24 & 44 & 17 & 39 \\
\hline 20 & 13 & 23 & 18 & 27 & 19 & 45 & 16 & 39 \\
\hline 21 & 5 & 17 & 12 & 22 & 44 & 51 & 7 & 42 \\
\hline 22 & 5 & 18 & 32 & 24 & 21 & 62 & 5 & 33 \\
\hline 23 & 5 & 11 & 14 & 36 & 34 & 60 & 6 & 34 \\
\hline 24 & 19 & 26 & 26 & 26 & 3 & 41 & 20 & 39 \\
\hline 25 & 20 & 18 & 19 & 30 & 13 & 43 & 18 & 39 \\
\hline 26 & 2 & 9 & 9 & 15 & 65 & 48 & 10 & 42 \\
\hline 27 & 4 & 15 & 32 & 21 & 28 & 51 & 13 & 36 \\
\hline 28 & 4 & 19 & 41 & 24 & 12 & 56 & 11 & 33 \\
\hline 29 & 11 & 10 & 15 & 21 & 43 & 48 & 9 & 43 \\
\hline 30 & 16 & 22 & 19 & 23 & 20 & 48 & 8 & 44 \\
\hline 31 & 11 & 16 & 26 & 31 & 16 & 54 & 8 & 38 \\
\hline 32 & 3 & 19 & 35 & 28 & 15 & 66 & 2 & 32 \\
\hline 33 & 9 & 20 & 21 & 32 & 18 & 54 & 13 & 33 \\
\hline 34 & 10 & 26 & 42 & 18 & 4 & 56 & 12 & 32 \\
\hline 35 & 9 & 20 & 35 & 31 & 5 & 62 & 7 & 31 \\
\hline 36 & 20 & 22 & 20 & 30 & 8 & 48 & 14 & 38 \\
\hline Average & 13 & 21 & 26 & 24 & 16 & 51 & 12 & 37 \\
\hline
\end{tabular}


the neural net as valid data (i.e., containing one of the five driving scenarios). Column 8 shows the percentage of time no valid target was detected by the range sensor. Column 9 contains the percentage of time that range/range-rate data was either intermittent or not recognizable as any of the five driving scenario patterns. The average value for each column (across all 36 drivers) appears as the last row of Table 1 . Interestingly, none of the individual drivers comes particularly close to matching the profile computed by this average.

The real value provided by the neural net processing in all these examples is the efficiency, accuracy, and automation achieved for an otherwise tedious manual task of searching for and counting thousands of occurrences of specific driving behavior patterns contained within large volumes of recorded data. The performance of the neural net was checked in this application by manually verifying results on a subset of unseen data for ten drivers. False predictions and misses were encountered at a rate of about 2-3\%. That is, for 120 actual encounters occurring during an hour of driving, 1 or 2 were missed, and 1 or 2 were incorrectly classified. Training time for the network was on the order of a minute or two using a conventional desktop PC. Processing time for an hour of driving data was similarly brisk The most time (perhaps two days or so) was spent manually reviewing and selecting a broad variety of representative cases collected from several different drivers for use in performing the initial network training.

\section{COMPARISON OF RESULTS USING A DRIVING AGGRESSIVITY INDEX}

One way of comparing the above results in terms of differences between drivers, is to formulate an "aggressivity index" that reflects the willingness (or frequency of occurrence) of a particular driver to overtake and pass other vehicles. A simple example would be the following equation (1),

$$
A I=(C I R+C I)+F / 2
$$

where,
AI is the computed driver aggressivity index value
CIR is the "Closing-in rapidly" percentage (column 2 of Table 1)
$\mathrm{Cl}$ is the "Closing-in" percentage (column 3 of Table 1), and
$\mathrm{F} \quad$ is the "Following" percentage (column 4 of Table 1).

This equation is simply the sum of percentages 2 spent "closing-in" or overtaking other vehicles (regardless of the degree) plus half of the "following" percentage. All computed values then lie in the range from 0 to 1 . A driver who spends equal percentages of time (33.3\%) passing, following, and being passed by other vehicles

2 normalized by 100 
would have a value of 0.5 by this formula. Similarly, a driver who passes all vehicles and never follows or is passed by another vehicle has a value of 1.0 ; a driver who is passed by everyone has a value of 0 .

If this simple formulation is applied to the data appearing in Table 1 , the plot in Figure 8 shows the result of AI versus subject. As seen, index values range from 0.15 to 0.78 .

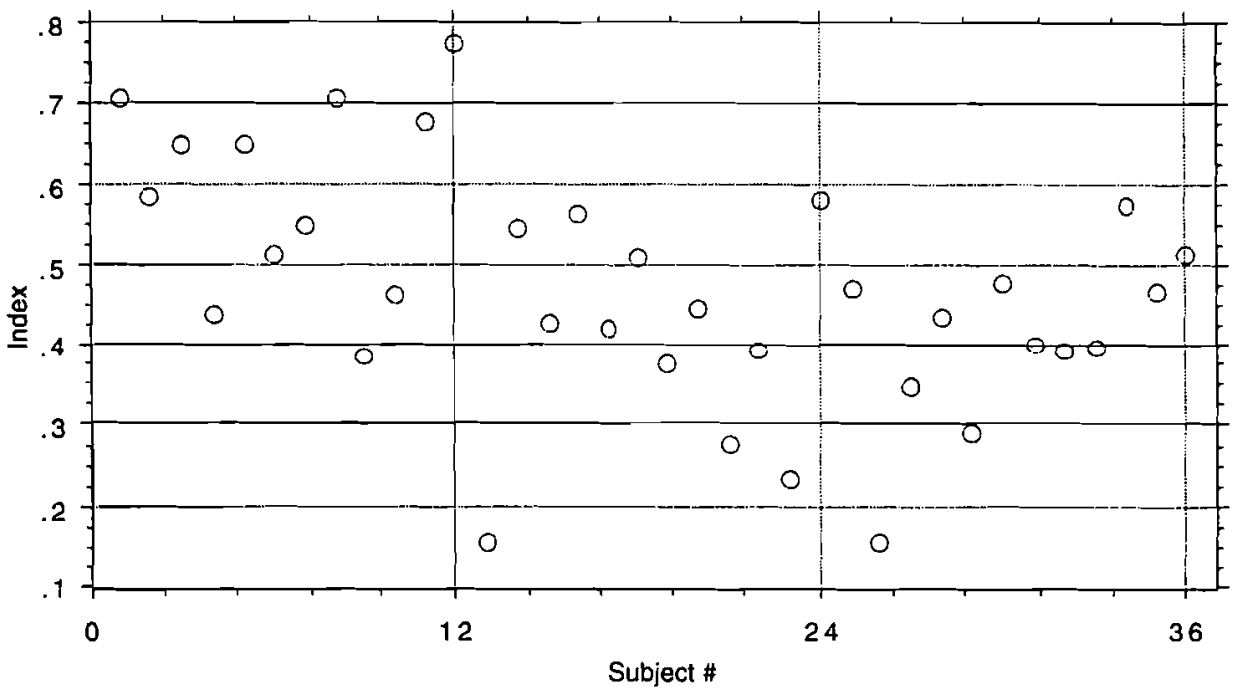

Fig. 8. Aggressivity index vs. subject as computed from equation (1).

The numbering system selected for the driving subjects happens to group them according to age. The youngest drivers (ages 20-30) have Subject numbers 1-12; middle-aged drivers (ages 40-50) have numbers 13-24; and older drivers (ages 60-70) have numbers $25-36$. If these subjects are now grouped by age and plotted, the results are seen in Figure 9.

As indicated in Figure 9, the group of younger drivers has a clearly higher average value and larger minimum and maximum AI values than either of the older groups. Very little differences are noted between the middle-aged and older driver groups.

A frequency distribution of the aggressivity index calculated by equation (1) for all drivers is seen in Figure 10 showing a mean value of 0.47 and standard deviation of 0.147. An overlay line of the corresponding normal distribution curve is also included for comparison. The only drivers appearing in the three highest bins of Figure 10 (Index values above 0.6 ) correspond to drivers from the younger group (6 drivers). 


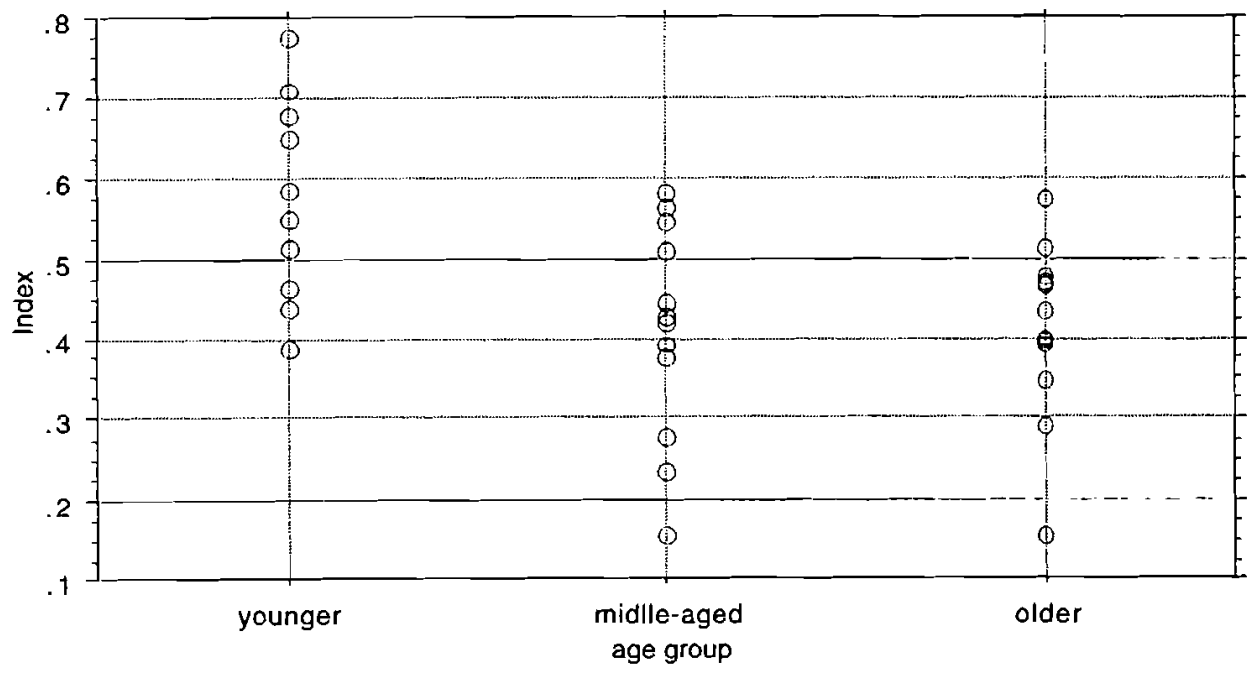

Fig. 9. Aggressivity index vs. age group.

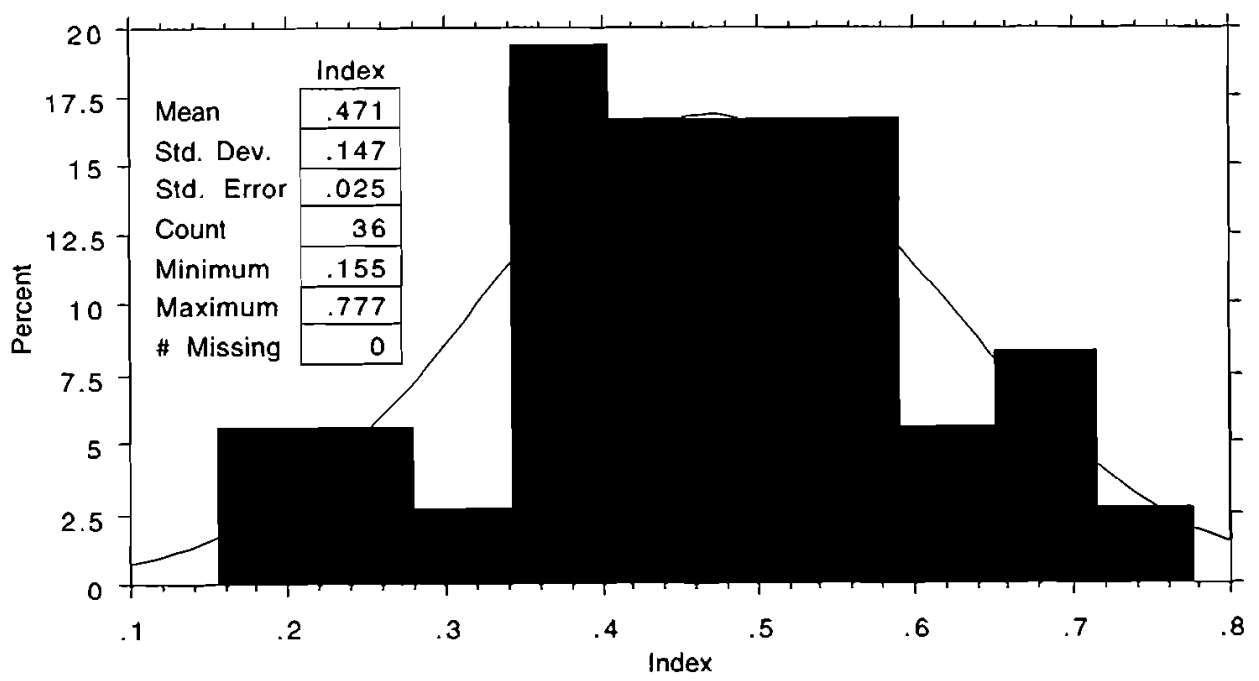

Fig. 10. Frequency distribution of the aggressivity index.

The results show a relatively normal distribution of aggressivity index values, further evidenced by the S-curve shape of the percentile plot in Figure 11. Both tails of this curve show two clusters of six drivers each at the low and high end of the Index range. The remaining 24 drivers distribute their values more or less uniformly across 
the Index range of 0.4 to 0.6 . The horizontal lines represent the $10 \mathrm{th}, 25 \mathrm{th}, 50 \mathrm{th}, 75 \mathrm{th}$, and 90 th percentile values. Four drivers lie in the upper 10th percentile; another four lie in the lower 10 th percentile.

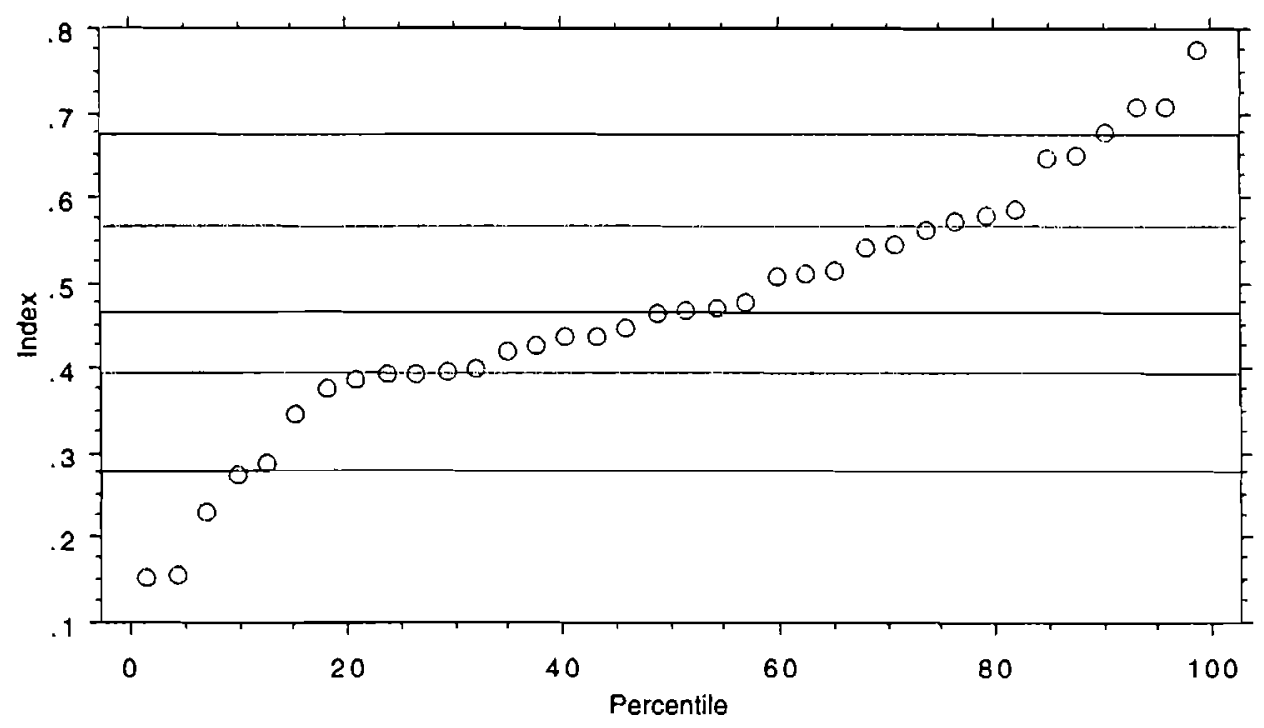

Fig. 11. Percentile plot of aggressivity index.

Results like these can then be used to help provide a profile of driver behavior or to describe expected distributions of driving behavior for various types of driving tasks. For headway control system applications, system parameters may be adjusted based upon the results of specific driver analyses.

\section{LONGITUDINAL CONTROL APPLICATIONS}

The prior sections of the paper have described a pattern recognition application of neural networks for identifying various categories of driving behavior based upon streams of input data containing range and range-rate information. This last section addresses the application area of control, and how neural nets may be used to represent driver manual control of throttle position during headway keeping tasks. Similar use of neural nets to represent steering control behavior of drivers has proven useful in prior applications and other control topics [5,7]. An example of using a neural net to represent the longitudinal control behavior associated with the closing-in and subsequent tracking of a preceding vehicle by one of the 36 drivers in this study is seen in Figures 12 - 14. Here, the accelerator pedal position is being represented as a function of range and range-rate (and their time-delayed counterparts) by a two-layer network architecture seen in Figure 12. The time delay parameter, $\tau$, is used to help the network calculate derivative-related information or transport delays through differencing operations. 
Values of $\tau$ used here were in the range of $1-2$ seconds. The calculated output is the accelerator pedal position seen in Figure 13. The corresponding range vs. range-rate diagram is seen in Figure 14.

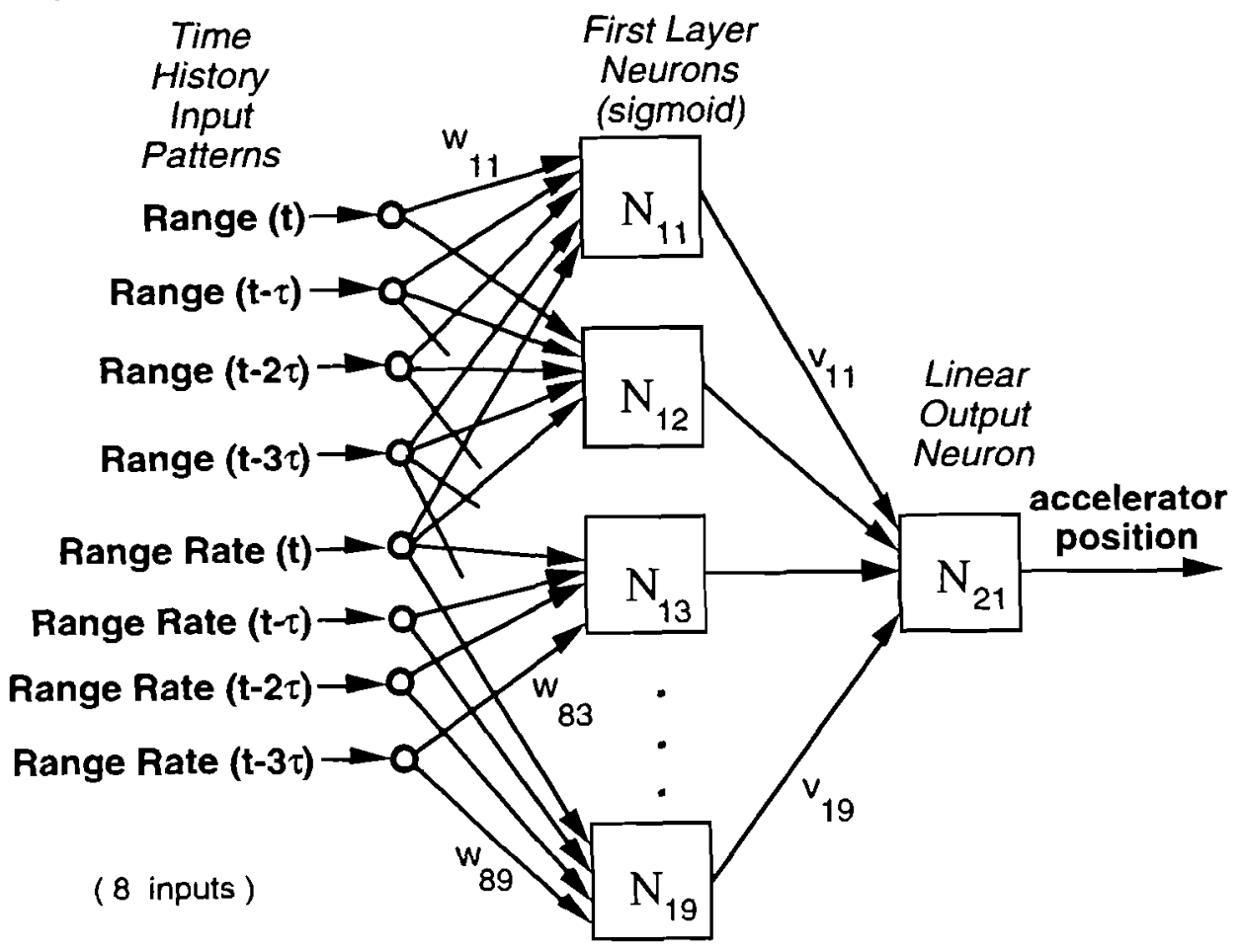

$w$ and $v$ are network weights calculated during network training

Fig. 12. Network architecture used in the longitudinal control example.

In this example, the driver is initially coasting in on a preceding target vehicle (accelerator off) and then begins to follow at a fairly constant headway. During the following portion, the driver is engaged in an on-off throttle modulation activity that is frequently observed of drivers during manual headway control operation. The bangbang nature of the control modulation suggests the likely presence of certain nonlinearities such as thresholding of sensed signals, lags in power train dynamics, and transport delays within the driver.

As indicated in Figure 13, the neural net representation produces a good replication of the measured driver accelerator control response during this longitudinal tracking maneuver. Similar results have also been obtained for comparable operating scenarios. 


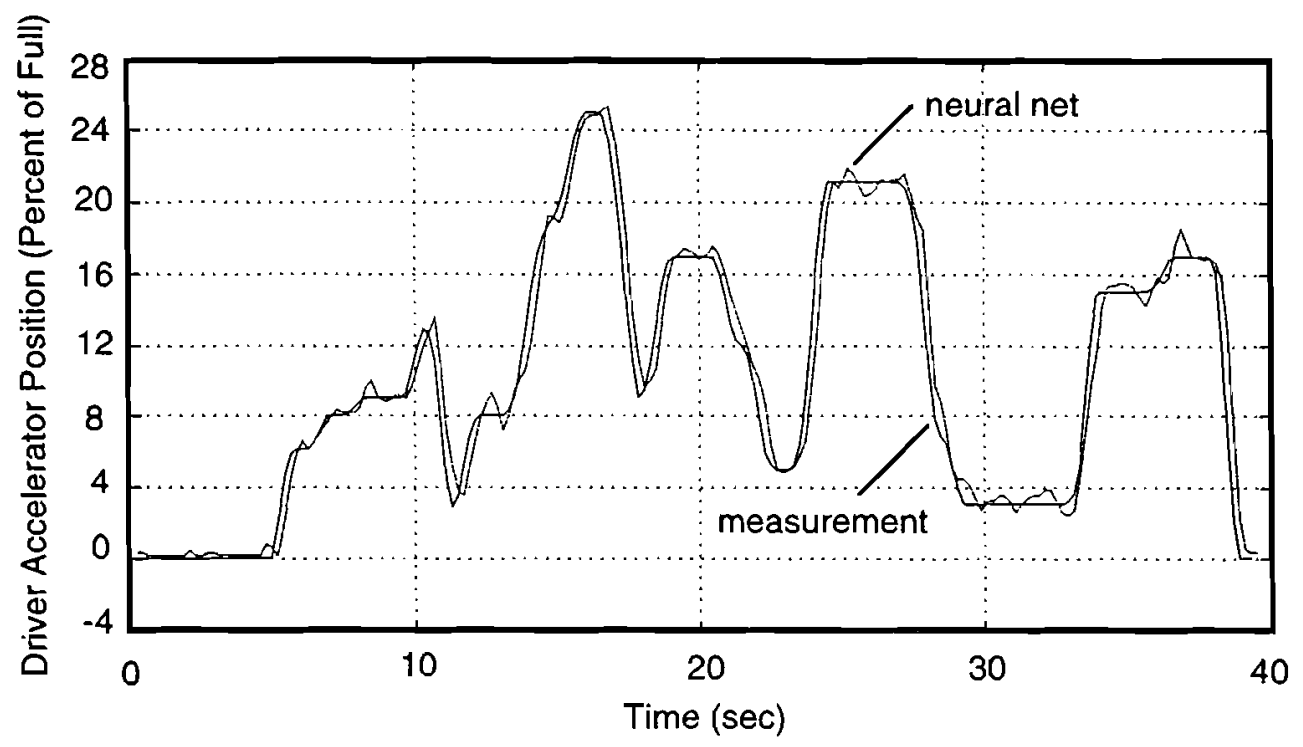

Fig. 13. An example result of representing driver longitudinal control behavior with a neural net.

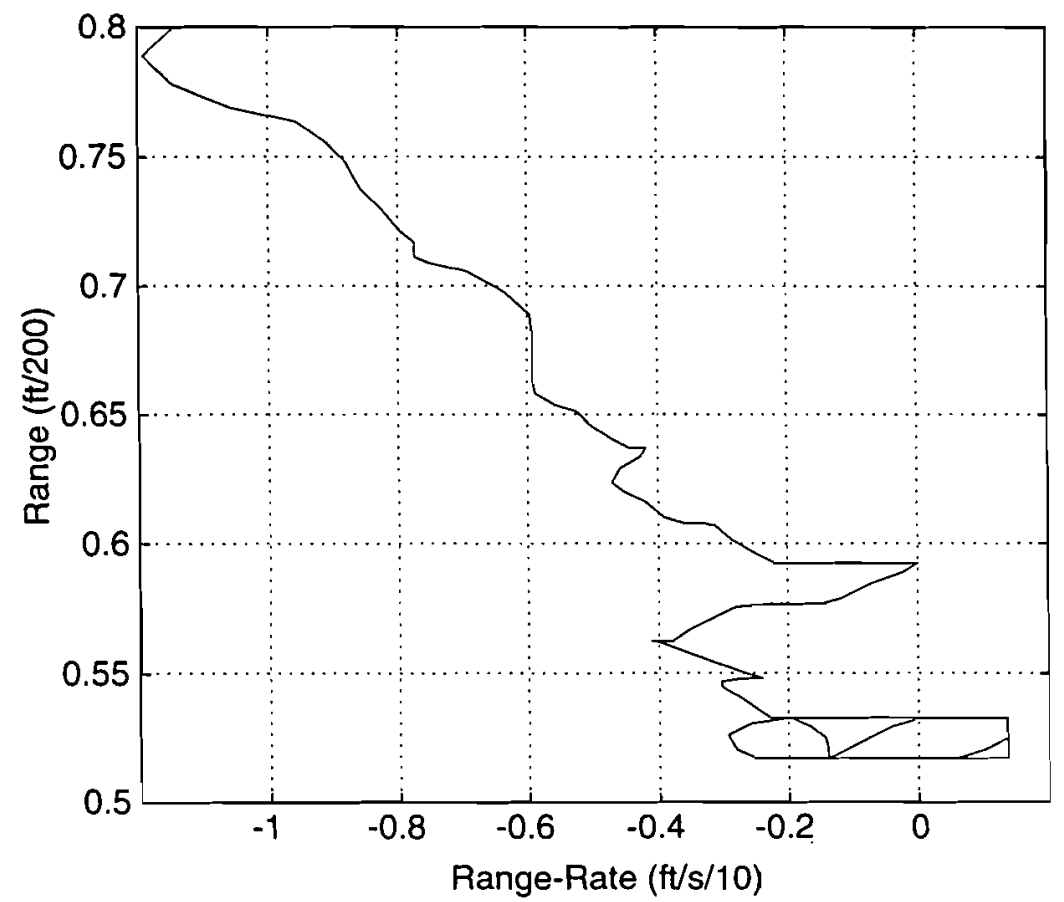

Fig. 14. Corresponding range vs. range-rate plot. 
However, use of any one identified neural net model (i.e., weights and architecture of Figure 12) corresponding to some specific data sample (such as that seen in Figures 13 - 14), does not necessarily produce comparable levels of agreement for new data samples collected under similar operating conditions a short time later with the same driver. That is, a neural net representation derived from prior training data does not necessarily predict future driver control behavior under similar operating conditions with the same degree of accuracy as reflected in Figure 13. Part of the explanation may be that the longitudinal control task as examined here is: 1) frequently affected by the driving environment such as road grade, nearby vehicles, visual distractions, and other influences that are not reflected directly within the limited sensor information provided by just range and range-rate measurements, and 2) a relatively casual control activity for most drivers not demanding perhaps the same level and continuity of attention that might be required for a task such as path-following. Consequently, drivers are likely affected by other influences beyond just range and range-rate - as assumed by the simple longitudinal control formulation offered here. Road grade, for example, has a significant influence on the dc-component of the accelerator position required to accomplish the same tracking task. A more robust control formulation would therefore include this type of influence (through longitudinal accelerometer measurements) and thereby extend the likely range of application of any such control model.

At present, UMTRI is examining these types of issues to help understand and further the potential use of neural networks in the area of adaptive headway control applications. A subsequent paper will report on those findings.

\section{CONCLUSIONS}

A neural network application for pattern recognition has proven useful for streamlining data analyses associated with the categorization of driving behaviors exhibited by a group of 36 test subjects. An "aggressivity index" that reflects a driver's observed willingness to pass other vehicles was formulated using the results from the neural net analyses. The aggressivity index helped to describe distributions of passing behavior exhibited by the group of 36 drivers as well as differences between individual drivers.

In a second application, a neural network was used to model longitudinal control behavior associated with closing-in and subsequent tracking of a preceding vehicle. Initial results demonstrate the ability to model specific instances of driver control behavior. However, further research is being pursued to help extend the predictive capabilities (and accompanying utility) of such models beyond the specific operating conditions for which they are identified.

Example applications of these basic technologies could potentially include: 1) onboard real time processing systems for obtaining adaptive and updated characterizations of driver control behavior (for tuning headway control system properties or monitoring driver control performance), and 2) development of headway control algorithms that are 
more closely aligned with driver expectations of how such systems should operate or feel.

\section{ACKNOWLEDGEMENT}

Much of this work was supported by the U.S. Department of Transportation and the National Highway Traffic Safety Administration under the Cooperative Agreement entitled "Fostering the Development, Evaluation, and Deployment of Forward Crash Avoidance Systems (FOCAS)," No. DTNH22-94-Y-47106.

\section{REFERENCES}

[1] Fancher, P. et al., "Evaluation of Human Factors and Safety Performance in the Longitudinal Control of Headway," Proceedings Of the Second World Congress on ITS, Yokohama, Japan, 1995.

[2] Fancher, P. et al, Fostering Development, Evaluation, and Deployment of Forward Crash Avoidance Systems (FOCAS), U.S. DOT Annual Research Report ARR-12-1596, UMTRI-96-44, December 1996.

[3] Sayer, J., Mefford, M., and Fancher, P., "Consumer Acceptance of Adaptive Cruise Control Following Experience with a Prototype System," Proceedings of the Human Factors and Ergonomics Society 39th Annual Meeting, Santa Monica, CA 1995.

[4] Turrentine, T., Sperling, D., and Hungerford, D., "Consumer Acceptance of Adaptive Cruise Control and Collisions Avoidance Systems," Transportation Research Record, No. 1318, 1991, pp. 118-121.

[5] MacAdam, C.C. and Johnson, G. E., "The Application of Elementary Neural Networks and Preview Sensors for Representing Driver Steering Control Behavior," Vehicle System Dynamics, Vol. 25, No. 1, 1996.

[6] Looney, C.G., Pattern Recognition Using Neural Networks, Oxford University Press, New York, 1997.

[7] Miller, W.T., Sutton, R.S., and Werbos, P.J. editors, Neural Nerworks for Control, MIT Press, Cambridge, Massachusetts / London, 1995. 